Full length article

\title{
Photonic millimeter-wave bridge for multi-Gbps passive optical networks
}

\author{
Ivan Aldaya ${ }^{a, *}$, Carolina Del-Valle-Soto ${ }^{b}$, Gabriel Campuzano ${ }^{c}$, Elias Giacoumidis ${ }^{\text {, }}$, \\ Rafael González ${ }^{\mathrm{C}}$, Gerardo Castañón ${ }^{\mathrm{C}}$ \\ a Sao Paulo State University, Campus Sao Joao da Boa Vista, SP, Brazil \\ b Universidad Panamericana, Facultad de Ingeniería, Prolongación Calzada Circunvalación, Poniente 49, Zapopan, Jalisco, 45010, Mexico \\ c Engineering School, Tecnologico de Monterrey, Mexico \\ d Dublin City University, School of Electronic Engineering, Dublin 9, Ireland
}

\section{A R T I C L E I N F O}

\section{Article history:}

Received 11 September 2017

Received in revised form 16 February 2018

Accepted 2 April 2018

Available online 7 April 2018

\section{Keywords:}

Passive optical networks

mm-wave links

Network survivability

\begin{abstract}
A B S T R A C T
Survivability is a critical requirement of optical communication networks that is typically addressed implementing path diversity. However, due to the elevated cost of fiber installation, this approach may prove prohibitively expensive in optical access networks. In this paper, a novel cost-efficient photonic millimeter $(\mathrm{mm})$-wave bridge is proposed, which converts passive optical network (PON) signals to radiofrequency signals at mm-wave bands. The performance of the mm-wave photonic bridge is numerically tested, revealing its feasibility to transmit a 2.5-Gbps PON with $-55.6 \mathrm{~dB}$ wireless link gain (WLG) using the 81 $86 \mathrm{GHz}$ band and $10-\mathrm{Gbps}$ PON with $-35 \mathrm{~dB}$ at the $102-109.5 \mathrm{GHz}$ band. The effect of fiber is also analyzed, showing that fiber cuts closer to the optical network unit degrades more the system performance.
\end{abstract}

(c) 2018 Elsevier B.V. All rights reserved.

\section{Introduction}

Optical fiber has emerged as the only transmission technology capable of meeting the increasing capacity requirement not only in long-haul links, but also in metropolitan and access networks [1]. In this context, passive optical networks (PONs), where the signal is transmitted through an optical distribution network without amplification, have emerged as a cost-efficient technology and are being deployed all around the world to offer higher transmission rates to the end user [2]. PON standards have evolved to adapt to user demands [3], from the obsolete 155-Mbps broadband PON standard to Gigabit PON (G-PON) [4] and state-of-the-art XGPON [5] operating at 2.5 and $10 \mathrm{Gbps}$, respectively. Furthermore, PON became a key enabling technology supporting backhaul in already deployed 3G cellular systems and future 4G and 5G systems [6]. However, deployment of PON networks is sometimes complicated due to urban architecture or geographical constraints, specially in vast countries such as Australia or Mexico. In addition, PON networks are very sensitive to seismic and other natural catastrophes as shown by the devastating earthquake in Japan in 2011 [7,8]. Given the dependency of most services on internet connection, this earthquake revealed a critical Achilles' heel in existing optical networks, including those supporting the emergency management.

\footnotetext{
* Corresponding author.

E-mail address: ivan.aldaya@sjbv.unesp.br (I. Aldaya).
}

Survivability is a key feature of any network design, but in the particular case of optical networks, is even more important due to the great amount of transmitted data and the difficult fiber repair process. In long-haul optical links, vulnerability to accidental fiber cuts is addressed through self-healing topologies, such as ring-based schemes or spatial diversity [9]. But these approaches may result prohibitively expensive in optical access networks where the cost associated to fiber installation represents the mayor part of the implementation expenses [10,11]. An attractive alternative consists on using a wireless bridge, that is, a point-to-point wireless connection, communicating two fiber spans $[7,8,12,13]$. Because of the high bitrate of the transmitted signal, this wireless bridge can only operate at millimeter-wave (mm-wave) frequencies, where huge unlicensed bands are still underutilized [14]. In [12], electrical means are used to generate a signal at $220 \mathrm{GHz}$ suitable for radiation, whereas in [7] and in [13], the signal is generated in a centralized fashion using a typical radio over fiber (RoF) approach [15]. The former offers the possibility to change the properties of the signal, optimizing and adapting the modulation format to the wireless channel, but it requires high-frequency components and electronics with broadbandwidth signal processing capabilities, increasing not only the cost but also the power consumption. In addition, the electrical demodulation, modulation, and up-conversion processes induce a time latency that may degrade the transmission performance. On the other hand, the RoF approach requires lower bandwidth electronics and causes minimal latency, but it lacks control on 


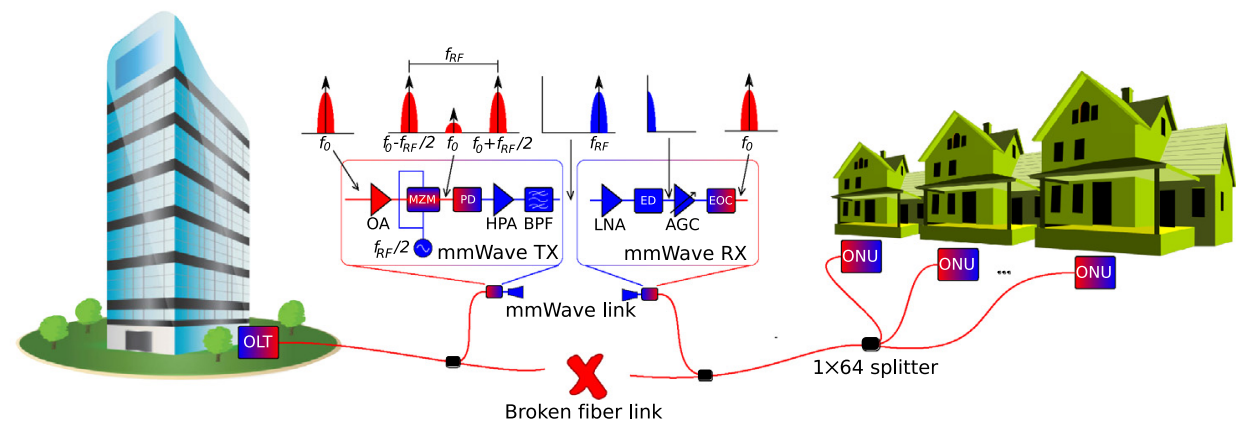

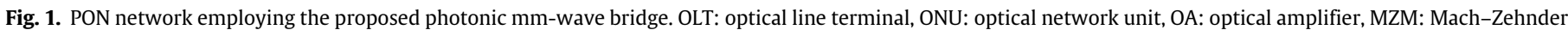

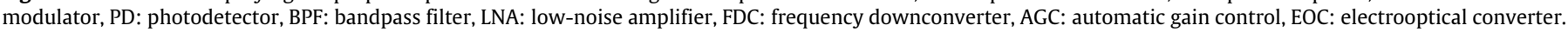

the modulation format of the radiated signal and, since there is not signal regeneration, the effects of the impairments both in the fiber and wireless channels are accumulated. In addition, in the schemes presented in [7] and in [8], as in many RoF schemes, the high-frequency RF signal is generated by photo-detecting an optical signal that has a bandwidth much broader than that of a typical PON signal. Since this signal is generated in the ONT, it must traverse all the optical network up to the fiber cut, which may result in bandwidth incompatibility. Even more, since the RoF signal has to travel from the ONT to the fiber cut, it is affected by the fiber chromatic dispersion.

In this paper we present a novel photonic mm-wave bridge based on RF frequency doubling, which is shown in Fig. 1. The incoming optical signal is amplified and remodulated using a MachZehnder modulator (MZM) before being detected by a broadbandwidth photodetector (PD). Afterwards, the photogenerated signal is amplified using a high-power amplifier (HPA) and radiated. In the wireless receiver, the mm-wave signal is downconverted to baseband and converted back to the optical domain. In comparison to [12], the number of high-frequency devices is significantly lower and the required frequency of the oscillator is reduced by a factor of two thanks to the RF frequency doubling using optical means. On the other hand, compared to [7] and [13], the proposed scheme is less sensitive to fiber chromatic dispersion because the generated RoF signal is not transmitted over a long fiber span up to the fiber cut (mm-wave bridge). In addition, the network is modified only at the two sides of the fiber cut, leaving the rest of the network unaltered, which ensures compatibility with the previously implemented network. However, the feasibility of the proposed scheme is still uncertain, as well as the wireless range achievable and the maximum fiber spans that can be connected. In order to elucidate these points, the proposed scheme has been tested using VPI Transmission Maker, which has been fed with realistic parameters obtained from commercial data sheets. Numerical results reveal the potential of this approach not only as an emergency link but also as early deployment or low-cost infrastructure for both G-PON and XG-PON. A BER below $10^{-9}$ has been obtained for G-PON NRZ-OOK transmission using the 81-86 $\mathrm{GHz} \mathrm{mm}$-wave band with a minimum required wireless link gain (WLG) of $-55 \mathrm{~dB}$ and XG-PON in the $102-109.5 \mathrm{GHz}$ band with a WLG of $-35 \mathrm{~dB}$. Simulation results also show that fiber cuts closer to the ONU degrade more significantly the system performance, causing a penalty in the required WLG.

The paper is organized as follows: Section 2 describes in more detail the proposed mm-wave bridge, the PON signals to be transmitted, and the potential mm-wave bands. The simulation setup is introduced in Section 3, paying special attention to the electrical components. In Section 4, numerical results are presented and discussed, and Section 5 concludes the paper.

\section{Photonic mm-wave bridge and PON signals}

This section is divided into three subsections: first, the novel photonic mm-wave bridge is described. A second subsection briefly explains the PON signals that are considered in this work, whereas a third subsection is dedicated to the discussion on the potential mm-wave bands for each PON standard.

\subsection{Photonic mm-wave bridge architecture}

A network implementation based on the proposed photonic $\mathrm{mm}$-wave bridge is sketched in Fig. 1. The downlink PON signal is generated at the optical line terminal (OLT) and transmitted to the optical network units (ONU) using a passive distribution network that includes a first fiber span, a $1 \times 64$ splitter, and a second fiber span. In PON networks, the fiber span connecting the OLT and the splitter is specially critical because, if it is sectioned, all the ONUs served by the OLT run out of service.

Therefore, if the physical continuity of the first fiber is compromised because of an incident or if geographical constraints hamper its implementation, the mm-wave bridge can be used to create an alternative high-capacity wireless link. In the mmwave bridge transmitter, the power of the incoming PON signal is first optically amplified using a semiconductor optical amplifier (SOA). The signal is subsequently remodulated by a MZM, which is biased at zero-intensity point in order to perform RF frequency doubling by optical means, thus relaxing the required modulation bandwidth by a factor of 2 [16]. In this way, after the MZM, two sidebands separated by the desired RF frequency are obtained. These sidebands are photodetected using a broad-bandwidth PD. Once in the electrical domain, the signal is amplified by a HPA and radiated employing a highly directive antenna.

At the mm-wave receiver, the signal is amplified by a low-noise amplifier (LNA) before being downconverted using, for instance, an envelope detector (ED). Afterwards, a gain-controlled amplifier (GCA) conditions the signal strength to get the optimum power level for the electro-optical converter. The electro-optical conversion can be accomplished either by directly or externally modulating a diode laser. After converted back to the optical domain, the signal is transmitted to the ONU.

\subsection{PON signals}

In this work, two different PON signals are considered: (i) GPON, corresponding to the ITU-T G984 standard [4], operating at bit rates of 2.488 and $1.244 \mathrm{Gbps}$ for the downlink and uplink, respectively, and (ii) XG-PON, ITU-T G.987 [5], with 10-Gbps downlink and 2.5-Gbps uplink. Even if recently deployed PON networks are mainly XG-PON, we also study the case of G-PON since a part of the installed PON infrastructure still follows this standard. In both 
Table 1

Allowed bands for G-PON and XG-PON.

\begin{tabular}{|c|c|c|c|c|c|c|c|c|c|c|c|c|c|c|c|}
\hline \multirow[t]{2}{*}{ PON standard } & \multirow[t]{2}{*}{ Bitrate (Gbps) } & \multicolumn{2}{|c|}{$\mathrm{BW}(\mathrm{GHz})$} & \multicolumn{2}{|c|}{$57-64 \mathrm{GHz}$} & \multicolumn{2}{|c|}{$71-76 \mathrm{GHz}$} & \multicolumn{2}{|c|}{$81-86 \mathrm{GHz}$} & \multicolumn{2}{|c|}{$92-94 \mathrm{GHz}$} & \multicolumn{2}{|c|}{$94.1-100 \mathrm{GHz}$} & \multicolumn{2}{|c|}{$102-109.5 \mathrm{GHz}$} \\
\hline & & DSB & $\overline{\text { SSB }}$ & $\overline{\mathrm{DSB}}$ & SSB & DSB & $\overline{\text { SSB }}$ & $\overline{\mathrm{DSB}}$ & $\overline{\text { SSB }}$ & DSB & SSB & DSB & SSB & DSB & SSB \\
\hline G-PON (G.984) & 2.5 & 3.75 & 1.88 & $\checkmark$ & $\checkmark$ & $\checkmark$ & $\checkmark$ & $\checkmark$ & $\checkmark$ & $x$ & $\checkmark$ & $\checkmark$ & $\checkmark$ & $\checkmark$ & $\checkmark$ \\
\hline XG-PON (G.987) & 10 & 15 & 7.5 & $x$ & $x$ & $x$ & $x$ & $x$ & $x$ & $x$ & $x$ & $x$ & $x$ & $x$ & $\checkmark$ \\
\hline
\end{tabular}

cases, the optical signal is amplitude-modulated employing nonreturn-to-zero (NRZ) on-off keying (OOK).

NRZ-OOK is, from the point of view of the occupied bandwidth, the most challenging format. For the same bit rate, modulation formats with higher spectral efficiency may offer more flexibility in the selection of the mm-wave band and, consequently, an extra degree of freedom to choose the optimum electronics. It is envisaged, then, that the presented mm-wave bridge will be also capable of operating with emerging PON proposals with spectrallyefficient modulation formats, e.g. direct detection orthogonal frequency division multiplexing (DD-OFDM) [17]. In regards to PON systems with wavelength division multiplexing (WDM) [2], the proposed bridge could be upgraded to create multiple links by taking advantage of space multiplication in the wireless domain. The performance of the bridge in PON networks using a more advanced modulation formats and WDM are left as future work.

\section{3. mm-wave band selection}

There are several unlicensed bands in the mm-wave region that are candidate to accommodate such high-capacity RF signal [14]: the well-known $60-\mathrm{GHz}$ band ranging from 57 to $64 \mathrm{GHz}$ (in Europe and USA), two 5-GHz bands around 73.5 and $83.5 \mathrm{GHz}$, a relatively narrow 2-GHz band at $91 \mathrm{GHz}$, a 5.9-GHz band between 94.1 and $100 \mathrm{GHz}$, and a continuous 7.5-GHz band centered at $107.25 \mathrm{GHz}$. These unlicensed bands impose bandwidth constraints on the generated RF signals that, alongside with the frequency dependency of the electronics performance and the WLG, constitute important factors in choosing the optimum transmission band.

The bandwidth of the radiated RF signal depends on the optical bandwidth, and whether the photogenerated signal is filtered to generate a single sideband (SSB) or double sideband (DSB). In particular for, NRZ-OOK optical signals that results in electrical ASK signals, the DSB and SSB 3-dB bandwidths of the radiated signal are given by:

$$
\begin{aligned}
\mathrm{BW}_{D S B} & =1.5 \cdot R \\
\text { and } & \\
\mathrm{BW}_{S S B} & =0.75 \cdot R,
\end{aligned}
$$

where $R$ represents the bitrate of the transmitted signal. Therefore, the DSB bandwidth of G-PON is $3.75 \mathrm{GHz}$ and the SSB bandwidth is $1.875 \mathrm{GHz}$, whereas for XG-PON, the DSB and SSB bandwidths are 15 and $7.5 \mathrm{GHz}$, respectively. Since the bandwidth occupied by $\mathrm{G}-\mathrm{PON}$ is half the occupied by XG-PON, the latter is more difficult to be accommodated in the unlicensed bands.

Table 1 presents the allowable bands for the wireless transmission of both G-PON and XG-PON, considering DSB and SSB transmission. It is clear that, a G-PON signal can be converted to the electrical domain and transmitted in any of the unlicensed bands. It is even unnecessary to resort to SSB, except in the 90$92 \mathrm{GHz}$ band. In XG-PON systems, there is not any band capable of accommodating DSB transmission and only one band satisfies the bandwidth requirement, the $102-109.5 \mathrm{GHz}$ band, while the 57-64 GHz almost meets this criterion. In both G-PON and XGPON systems is not clear a priori what is the optimum band, but it requires simulations.

\subsection{Performance metrics}

In order to assess the performance of the system based on the proposed mm-wave bridge we will employ the bit error rate (BER). This is an intuitive metric that is usually estimated by two methods: it can be calculated by error counting comparing the transmitted and the received bit sequences. For low BER values, however, this method requires a large number of bits to be simulated. An alternative estimation approach for binary signals is the so-called $Q$-factor method, where BER is estimated by calculating the average and standard deviation values of the high and low levels. The $Q$-factor method is useful in systems limited by additive noise as in our case where the main source of noise is the noise induced by the electrical components of the bridge. The $Q$-factor is directly related not only to the BER but also to the signal-tonoise ratio (SNR) which is another common metric in noise limited systems. The $Q$-factor is related to the SNR through [18]:

$$
Q=\frac{1}{\sqrt{\mathrm{SNR}}}
$$

and, therefore, BER can be expressed both in terms of the $Q$-factor or SNR as:

$\mathrm{BER}=\frac{1}{2} \operatorname{erfc}\left(\frac{Q}{\sqrt{2}}\right)=\frac{1}{2} \operatorname{erfc}\left(\frac{1}{\sqrt{2 \cdot \mathrm{SNR}}}\right)$,

where erfc is the complimentary error function.

Even if BER is a useful metric for the overall system, it may not be the best choice to characterize the noisy bridge. For that purpose, in Section 4.4, we use another metric, i.e. noise figure (NF), which accounts for the SNR degradation when the signal passes through a noise device. Thus, NF can be calculated as:

$\mathrm{NF}(\mathrm{dB})=10 \log _{10}\left(\frac{\mathrm{SNR}_{\mathrm{in}}}{\mathrm{SNR}_{\text {out }}}\right)$,

where $\mathrm{SNR}_{\text {in }}$ and $\mathrm{SNR}_{\text {out }}$ are the SNR (in linear scale) at the input and output, respectively.

\section{Simulation setup}

The technical feasibility of the proposed architecture was tested using VPI TransmissionMaker 9.2. Fig. 2 shows the simulation setup alongside with some sampling spectra at different points of the system.

In the OLT, a $2^{14}$-bit pseudorandom binary sequence (PRBS) was generated in each run and coded into an electrical signal using NRZ format with a bit rate of 2.5 (10) Gbps for PON (XG-PON). A secondorder low-pass filter with a bandwidth of 0.75 times the bit rate of the signal was used consider the bandwidth limitation of the OLT electrical equipment and to limit the occupied bandwidth.

The signal was then converted to the optical domain using direct modulation (G-PON), or external modulation (XG-PON). The directly modulated laser was modeled using the rate equations that relate the complex field and carrier density within the singlemode laser cavity. With the parameters listed in Table 2, the laser exhibits a threshold current of $20 \mathrm{~mA}$ and a current slope of $0.3 \mathrm{~mW} / \mathrm{mA}$, which are reasonable values for commercial distributed feedback (DFB) lasers. The driving electrical signal was amplified and combined with a bias current resulting in a signal varying from 3 to 5 times the laser threshold current. On the other 


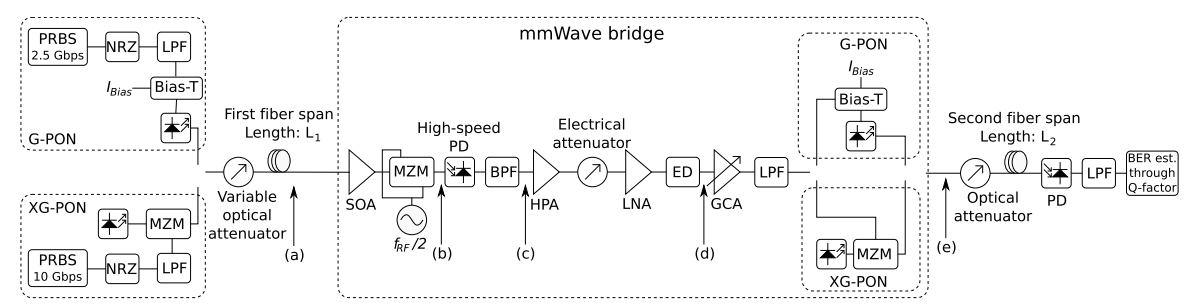

(a)
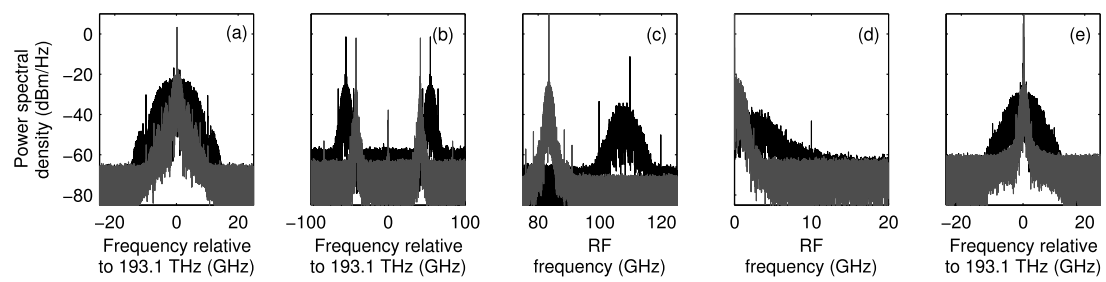

(b)

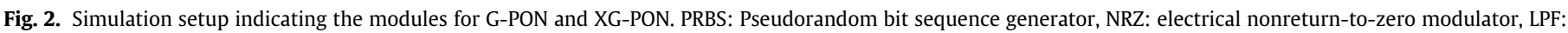

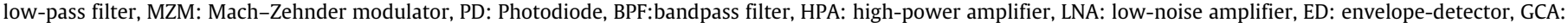

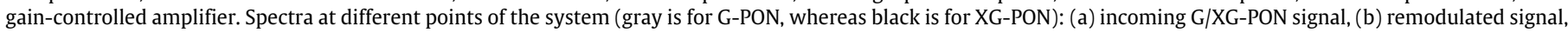
(c) generated mm-wave signal, (d) downconverted signal, and (e) transmitted G/XG-PON signal.

Table 2

Simulation parameters

\begin{tabular}{llll}
\hline & Parameter & Value & Unit \\
\hline \multirow{3}{*}{ General } & Bit rate & $2.5 / 10$ & $\mathrm{Gbps}$ \\
param. & Number of bits & $2^{14}$ & - \\
& Samples per symbol & 32 & - \\
& Time window & $13.1 / 3.28$ & $\mu \mathrm{s}$ \\
\hline \multirow{4}{*}{ Laser } & Nominal wavelength & 1550 & $\mathrm{~nm}$ \\
& RIN & -150 & $\mathrm{~dB} / \mathrm{Hz}$ \\
& Linewidth & 1 & $\mathrm{MHz}$ \\
& Threshold current & 20 & $\mathrm{~mA}$ \\
& Adiabatic chirp & 10 & $\mathrm{GHz} / \mathrm{W}$ \\
\multirow{5}{*}{ SSMF } & Slope efficiency & 0.3 & $\mathrm{~W} / \mathrm{A}$ \\
& Attenuation & 0.2 & $\mathrm{~dB} / \mathrm{km}$ \\
& Dispersion coef. & 16 & $\mathrm{ps} / \mathrm{nm} / \mathrm{km}$ \\
& Dispersion slope & 80 & $\mathrm{ps} / \mathrm{nm}^{2} / \mathrm{km}$ \\
& Kerr coefficient & 0.026 & $\mathrm{~nm} / \mathrm{W}$ \\
\multirow{2}{*}{ MZM } & Core area & 80 & $\mu \mathrm{m}^{2}$ \\
& Insertion loss & 4 & $\mathrm{~dB}$ \\
& Bias voltage & 2.5 & $\mathrm{~V}$ \\
\hline
\end{tabular}

hand, for XG-PON, a MZM with an extinction ratio of $40 \mathrm{~dB}$ and an insertion loss of $4 \mathrm{~dB}$ was used to externally modulate the output of a laser with constant driving current (5 times the threshold current). In order to maximize the modulation depth of the optical signal, the MZM was set at the quadrature point. The resulting optical average power levels were $12.58 \mathrm{dBm}$ and $7.85 \mathrm{dBm}$ for direct and external modulation, respectively.

The standard single mode fiber (SSMF) connecting the OLT with the bridge, and the bridge with the ONU, was simulated using the split step Fourier transform (Table 2), which has shown to realistically mimic the behavior of dispersive nonlinear systems [19]. The $1 \times 64$ splitter was modeled inserting a $20-\mathrm{dB}$ optical attenuator that accounts for the splitting loss as well as for other impairments such as insertion and connection losses. In addition, a variable optical attenuator (VOA) was included at the output of the OLT to control the launched optical power at the input of the first fiber span.

Regarding the mm-wave bridge components, the broadbandwidth MZM of the bridge had a 6-dB insertion loss and a 40$\mathrm{dB}$ extinction ratio. The SOA was configured in power clamped mode with an output power of $15 \mathrm{dBm}$, a maximum gain of 20 $\mathrm{dB}$, and a noise figure of $9 \mathrm{~dB}$. This ensures that the SOA does not enter into saturation and, consequently, no pulse distortion is expected. In addition, the short optical path from the SOA up to the photodetection within the bridge transmitter avoids that the SOA-induced chirp to be converted into amplitude modulation and the subsequent distortion in the pulse shape. The optical to electrical conversion was made employing a high-speed PD with a responsivity that depends on its electrical bandwidth (Table 3 ), a $20 \mathrm{pA} / \sqrt{\mathrm{Hz}}$ thermal noise current spectral density, and shot noise. The parameters of most of the electrical components highly depend on the mm-wave band, as can be seen in Table 3. The electrooptical conversion in the mm-wave bridge was accomplished in the same way as in the OLT, that is, using direct modulation for 2.5 Gbps and external modulation for $10 \mathrm{Gbps}$. In order to optimize the modulation index, the driving power input at the directly modulated laser or external modulator was controlled using a gain controlled amplifier (GCA), after which a low-pass filter with a bandwidth of 0.75 times the signal bitrate removed the out of band noise.

Finally, the signal was detected at the ONU, which is based on a conventional direct detection scheme. The PD in the ONU had a 10$\mathrm{GHz}$ bandwidth, a responsivity of $0.9 \mathrm{~mA} / \mathrm{mW}$, and a thermal noise current density of $10 \mathrm{pA} / \sqrt{\mathrm{Hz}}$. Since the target bit error rate is very low, $10^{-9}$, error counting was unfeasible and BER was estimated through the mentioned $Q$-factor method [18].

\subsection{Electrical components}

The high-frequency components, both amplifiers and EDs, are modeled using the parameters obtained from data sheets supplied by Millitech [20], which is a manufacturer that has a portfolio covering the different bands for both amplifiers and EDs. The broadbandwidth PD specifications are obtained from different vendors depending on availability and performance. For less than $90 \mathrm{GHz}$, we use the data sheet from Henrich Hertz Institute (HHI) [21], while for higher frequencies we select products from Finisar [22]. Table 3 lists the most important parameters for each component and frequency band. For the HPA, both the gain and the 1-dB 
Table 3

Parameters of the electrical components.

\begin{tabular}{|c|c|c|c|c|c|c|c|}
\hline Device & Parameter & $57-64 \mathrm{GHz}$ & $71-76 \mathrm{GHz}$ & $81-86 \mathrm{GHz}$ & $92-94 \mathrm{GHz}$ & $94.1-100 \mathrm{GHz}$ & $102-109.5 \mathrm{GHz}$ \\
\hline \multirow{4}{*}{ HPA } & Model & AMP-15-03100 & AMP-12-02280 & AMP-12-40100 & AMP-10-41030 & AMP-10-02580 & AMP-08-40110 \\
\hline & Gain (dB) & 21 & 22 & 29 & 27 & 16 & 20 \\
\hline & $1 \mathrm{dBCP}(\mathrm{dBm})$ & 17.5 & 15 & 27.5 & 30 & 15 & 18.5 \\
\hline & Consumption (W) & 1.875 & 1.875 & - & 24.5 & 1.125 & - \\
\hline \multirow{4}{*}{ LNA } & Model & LNA-15-03090 & LNA-12-02190 & LNA-12-02220 & LNA-10-03130 & LNA-10-02130 & LNA-08-03210 \\
\hline & Gain (dB) & 19 & 12 & 15.5 & 19 & 30 & 17 \\
\hline & $\mathrm{NF}(\mathrm{dB})$ & 4.8 & 5.5 & 3.8 & 4.6 & 5.5 & 8.0 \\
\hline & Consumption (W) & 0.45 & 0.375 & 0.9 & 0.375 & 0.75 & 0.3 \\
\hline \multirow{3}{*}{ ED } & Model & DET-15 & DET-12 & DET-10 & DET-08 & DET-08 & DET-08 \\
\hline & Sensitivity $(\mathrm{mV} / \mathrm{W})$ & 850 & 850 & 1000 & 1000 & 1000 & 1000 \\
\hline & Max. input $(\mathrm{dBm})$ & -10 & -10 & -10 & -10 & -10 & -10 \\
\hline \multirow{3}{*}{ PD } & Model & $\mathrm{HHI}$ & $\mathrm{HHI}$ & $\mathrm{HHI}$ & XPDV4120R & XPDV4120R & XPDV412xR \\
\hline & Responsivity (A/W) & 0.6 & 0.6 & 0.6 & 0.6 & 0.6 & 0.5 \\
\hline & Max. output (dBm) & 12 & 12 & 12 & 7.5 & 7.5 & 7.5 \\
\hline
\end{tabular}

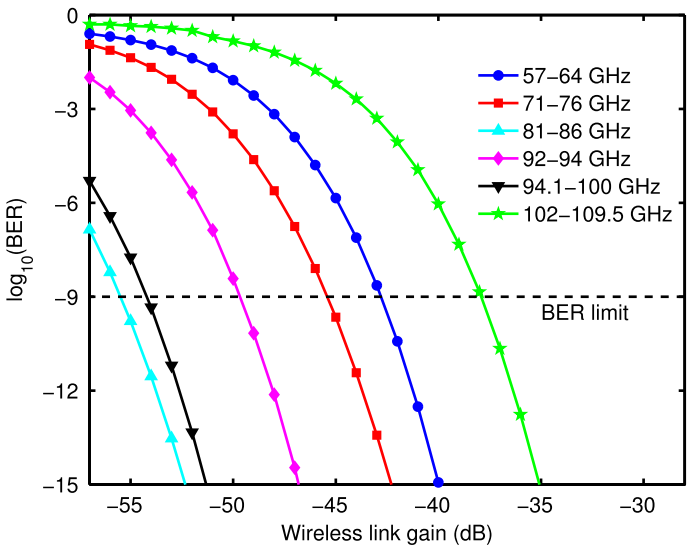

(a)

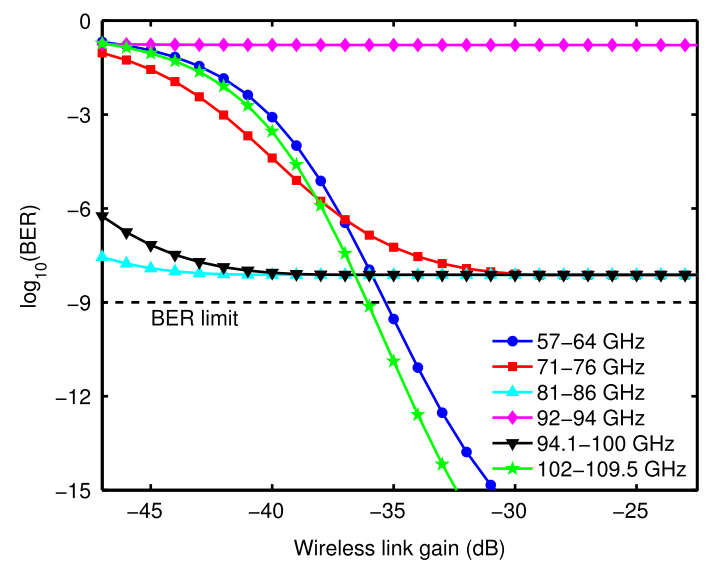

(b)

Fig. 3. BER in terms of the wireless link gain for the different mm-wave bands: (a) G-PON and (b) XG-PON.

compression point ${ }^{1}$ are given; whereas for the LNA, the gain and the noise figure are given. The reason for this difference is that the main impairment in the HPA is the nonlinear distortion while for the LNA the main nuisance is noise. Regarding the envelope detector, the key figure of merit is the sensitivity, which is typically expressed in $\mathrm{mV} / \mathrm{W}$.

\subsection{Wireless channel model}

Given the high operation frequency in the RF stage $(81-86 \mathrm{GHz}$ for G-PON and 102-109.5 GHz for XG-PON), we will assume a point-to-point directive line-of-sight (LoS) link with unblocked first Fresnel zone [23]. In clear terrestrial fixed links, reflections from any objects, including ground, present extremely low power and, consequently, there will not be multi-path effect. Additionally, since there is not moving scatterers within the line-of-sight and the relative position of the transmitter and receiver is fixed, no Doppler shift of spread are present. In this scenario, the LoS wireless link can be modeled as a flat attenuation, which is usually characterized by the wireless link gain (WLG). Generally, the WLG accounts not only for the path-loss but also for the transmitter and receiver effective antenna gains, $G_{T x}$ and $G_{R x}$ (it is worth noting that the term effective includes any penalty in the gain due to misalignment). We will present many of the results in terms of the WLG, which according

\footnotetext{
1 The output power at which the power level is reduced $1 \mathrm{~dB}$ from the linear response, often considered the maximum output power without significant distortion.
}

to the Friis formula can be calculated as:

$\left.\mathrm{WLG}\right|_{\mathrm{dB}}=\left.G_{T x}\right|_{\mathrm{dB}}+\left.G_{R x}\right|_{\mathrm{dB}}-\left.\operatorname{Loss}_{P a t h}\right|_{\mathrm{dB}}$.

It is important to note that we have neglected atmospheric losses since for the frequencies and distances we are considering freespace loss dominates [23]. To accomplish the WLG to distance conversion, we employed $40-\mathrm{dBi}$ antennas taken from the data sheet of Quinstar [24].

\section{Results and discussion}

In this section we first discuss the mm-wave band selection for G-PON and XG-PON. Afterwards, the photonic bridge is optimized for different received optical power levels and the effects of the fiber spans is analyzed for both standards. Finally, regression curves for the equivalent noise figure (NF) of the bridge are presented.

\subsection{Band selection}

According to Table 1, there are multiple choices to accommodate G-PON signals in the unlicensed mm-wave bands. The optimal selection was based on the performance of the electronic devices at different bands. Fig. 3(a) shows the BER in terms of the WLG for the different unlicensed bands in an optical backto-back configuration. The importance of band selection appears clear in this figure, showing a WLG difference between the best and worst bands of $17 \mathrm{~dB}$ at the BER threshold. Results reveal that the minimum required WLG is achieved in the $81-86 \mathrm{GHz}$ band, 


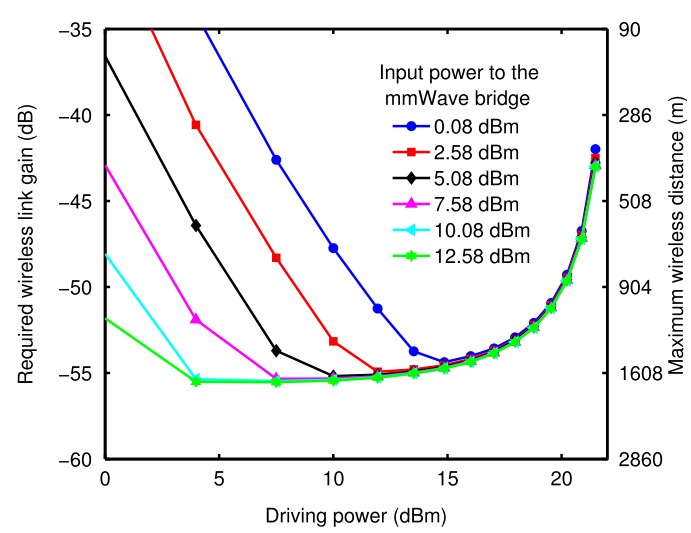

(a)

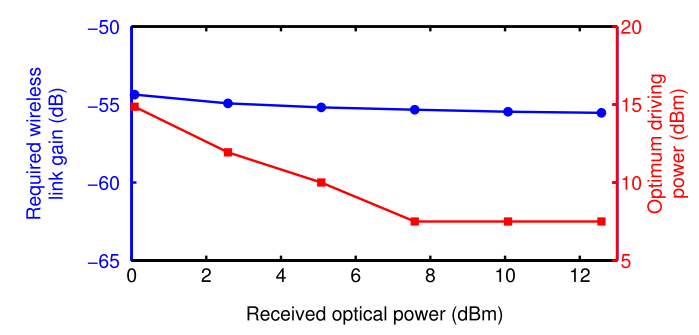

(c)

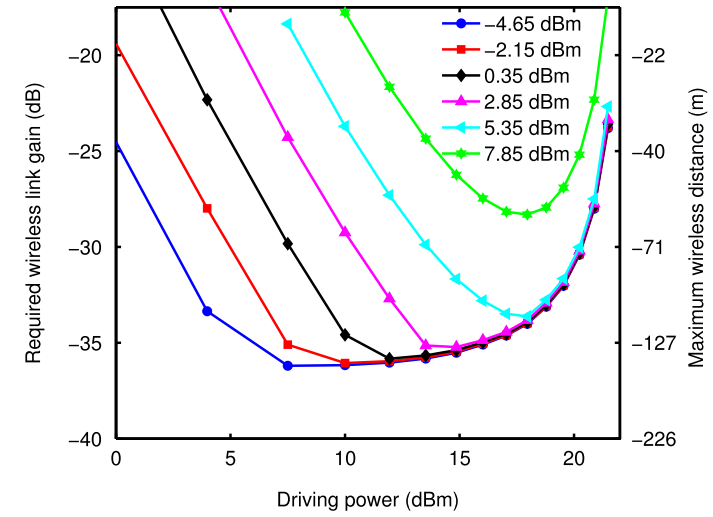

(b)

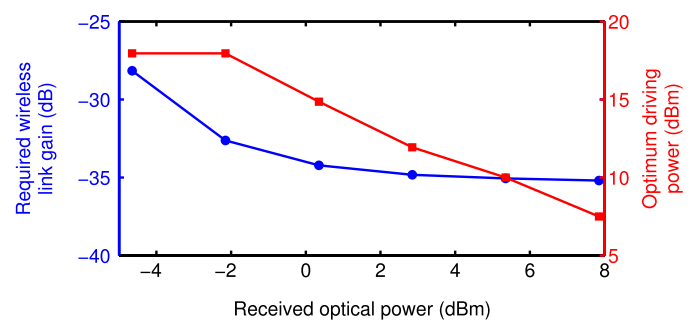

(d)

Fig. 4. Optimization of the driving power of the microwave tone at the MZM. Required wireless link gain to achieve a BER of $10^{-9}$ for different optical power at the input of the mm-wave bridge in terms of the driving power for (a) G-PON and (b) XG-PON. Required wireless link gain and optimum driving power for (c) G-PON and (d) XG-PON.

which is explained by the high performance of electronics in this band: high 1-dB compression point of the HPA, and low NF of the LNA.

Regarding XG-PON, band-limitation plays an important role. Recall that the 10-Gbps signal occupies a bandwidth of $7.5 \mathrm{GHz}$. So, as can be seen in Fig. 3(b), only the transmission at the $57-64 \mathrm{GHz}$ and 102-109.5 GHz bands achieves the required BER. This was an expected outcome since these bands are the widest. In the rest of the bands, an important percentage of the signal power is filtered out.

In conclusion, the optimum band for the transmission of the G-PON signal is the $81-86 \mathrm{GHz}$ band, whereas for XG-PON, the optimum mm-wave band is $102-109.5 \mathrm{GHz}$.

\subsection{Bridge optimization}

The noise added by the bridge components, such as the PD and amplifiers, is the main physical impairment that degrades the system performance. Its impact can be reduced by increasing the driving power of the MZM. However, given the nonlinear response of the MZM, a high driving power will induce nonlinear distortion. Therefore, the performance of the proposed architecture is ultimately limited by the combined effects of noise and nonlinear distortion.

Fig. 4(a-d) presents the required WLG and its corresponding maximum range as a function of the electrical driving power and the optical received power at the bridge. We used the VOA between the OLT and the bridge to sweep the received power, considering attenuations between 0 and $12.5 \mathrm{~dB}$ that approximately correspond to the loss of fibers of 0 to $60 \mathrm{~km}$. For both G-PON and XGPON, Fig. 4(a-b) shows that for low driving powers, the required WLG decreases at higher optical input powers, while for high driving powers, the required WLG is independent of the optical input power in G-PON; but for XG-PON, a $60 \mathrm{~km}$ fiber length increases the required WLG. Notice that the maximum wireless distance is an order of magnitude less for XG-PON.
In Fig. 4(c-d) the optimal driving power and required WLG are shown in terms of the received optical power. Comparing Fig. 4(c) and Fig. 4(d), we conclude that WLG suffers only a $1.5 \mathrm{~dB}$ penalty when decreasing the power from 8 to $-4 \mathrm{dBm}$ for G-PON. For XGPON this penalty increases to $7 \mathrm{~dB}$. These curves are employed in the next subsection to choose the driving power depending on the fiber length under study.

\subsection{Influence of the fiber cut position on the WLG}

The minimum WLG presented in Fig. 4(c) and Fig. 4(d) are for optical back-to-back, that is, without including optical fiber. In order to analyze the effect of both fiber spans, Fig. 5(a-f) show the BER curves for different combinations of fiber lengths. For GPON, the sequence of Fig. $5(\mathrm{a}-\mathrm{c})$ shows a progressive degradation of the system performance as the length of the first fiber span is increased. However, this is not the case in XG-PON where Fig. 5(de) show a minimal performance degradation if the length of the first fiber link is shorter than $30 \mathrm{~km}$. It is important to note that in both G-PON and XG-PON, the performance degrades much faster as the second fiber span length increases. This can be explained by the fact that in the second span, a 20-dB attenuator is included and, therefore, the system is more sensitive to losses in this span. The effect of the combination of different fiber span lengths can be appreciated more clearly in Fig. 6(a) and Fig. 6(b), where contour plots of the required WLG for G-PON and XG-PON are presented. As expected from Fig. 5(a-f), in G-PON, a longer first fiber span allows a shorter second fiber span to meet the BER requirement. In contrast, in XG-PON, the performance remains independent of the first fiber span up to $30 \mathrm{~km}$, after which, performance degrades significantly faster than in the case of G-PON. This behavior can be explained by noting that for short first spans, the generated $\mathrm{RF}$ power is limited by the output power of the SOA. Thus, it is independent of the attenuation in the first span and, consequently, of its length. For longer first span lengths, the RF power is limited by the gain of the SOA and the power and its input, leading to a dependence on the first fiber span length. 


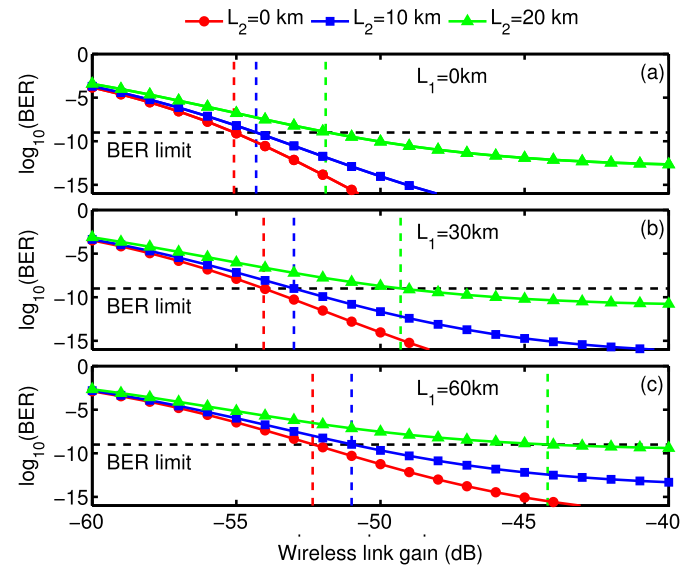

(a)

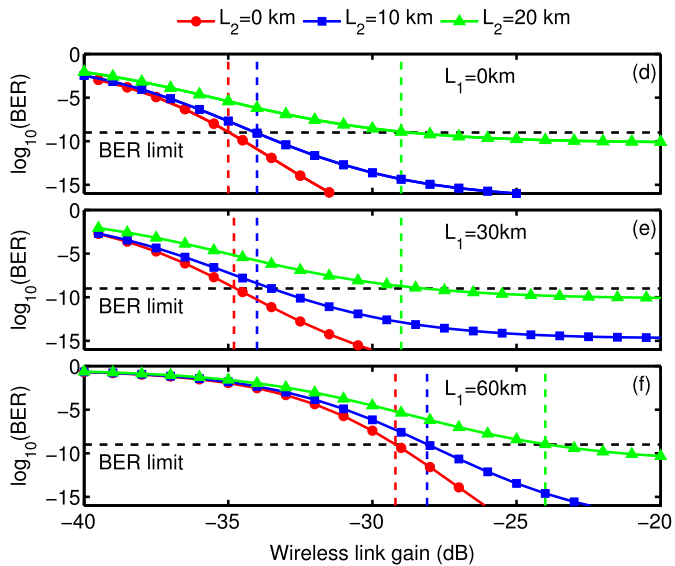

(b)

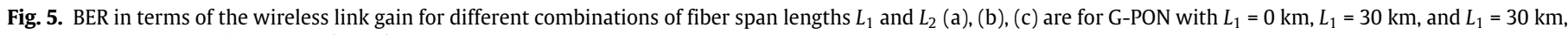
respectively, whereas (d), (e), and (f) are for XG-PON with the same $L_{1}$ values.

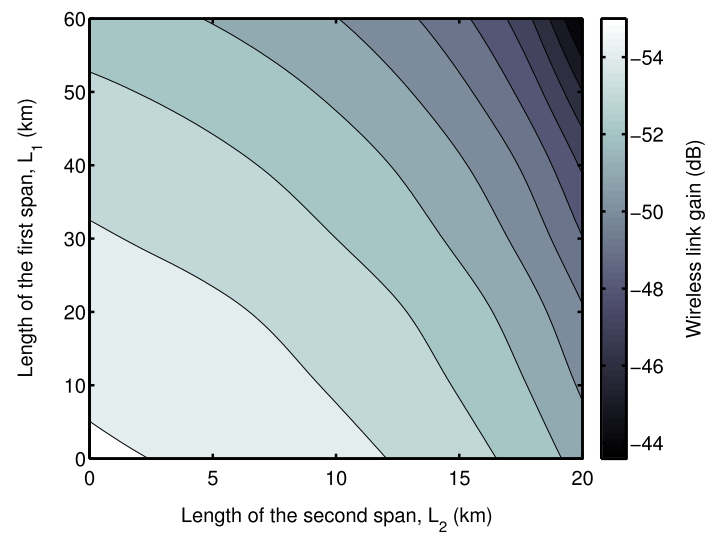

(a)

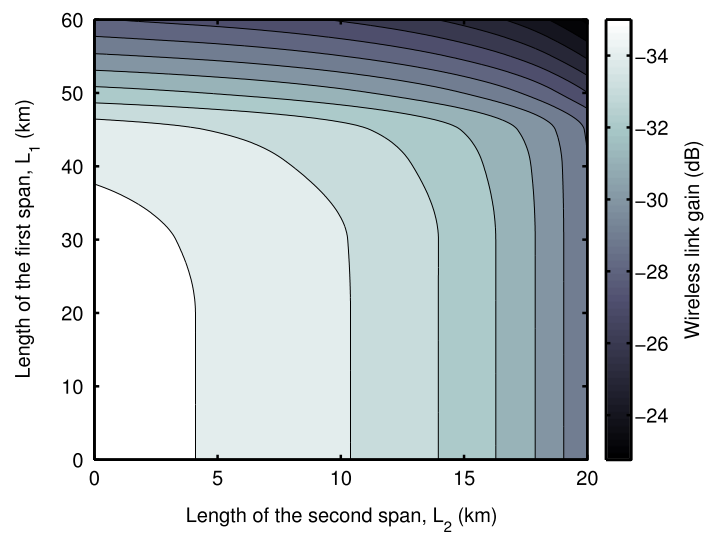

(b)

Fig. 6. Contour graphs for the minimum required wireless link gain to achieve a BER of $10^{-9}$ with different fiber lengths of $L_{1}$ and $L_{2}$ for (a) G-PON and (b) XG-PON.

\subsection{Modeling the bridge as a noisy power-clamped amplifier}

In this subsection, we propose a simplified model of the bridge, which has a twofold goal: first, to deep our knowledge of the signal degradation induced by the bridge and, second, to develop a model that can be used in a SNR budget analysis. Since the SNR depends on the signal and noise power, we need to model the output power and the amount of noise added by the bridge.

As explained in Section 2 the output power is fixed at the electrical-to-optical conversion stage. Hence, the bridge can be seen as a gain-controlled amplifier, or, equivalently, as an amplifier whose output power is clamped. The output powers are 12.58 and $7.85 \mathrm{dBm}$ for G-PON and XG-PON, respectively.

In regards to the added noise, we characterize it in terms of the noise figure (NF), which is defined as the difference in $\mathrm{dB}$ between the electrical SNR before and after the device under test [18]. ${ }^{2}$ It is expected that the NF will be highly dependent on the WLG since, at low values, the LNA will add significant noise, degrading the SNR at the output of the bridge. This assumption is supported by the curves presented in Fig. 7 for G-PON and XG-PON.

In both cases three different regions can be identified:

\footnotetext{
2 The conversion to the electric domain was performed using the same PD as in
} the ONU.
- For low WLG values, the NF is highly dependent on the WLG, which can be explained by noting that at low WLGs, the main noise source is the LNA.

- At high WLGs, the NF does not depend on the WLG. This can be attributed to the fact that, as the WLG increases, the received power at the mm-wave receiver also rises. Therefore, the LNA is not the main noise source any more and the thermal and shot noise of the PD dominate.

- For moderate WLGs, the SNR degradation due to the LNA and the PD are comparable. For these WLG values, the NF is not linear but may be approximated by a quadratic curve.

Although in both G-PON and XG-PON the three mentioned regions can be identified, the regression parameters in each region, as well as the boundaries between regions differ. For G-PON, the region where the main noise source is the LNA covers WLG up to $-56.1 \mathrm{~dB}$; while in $\mathrm{XG}-\mathrm{PON}$, this region is up to $-42.3 \mathrm{~dB}$. In addition, the slope is sharper in the case of XG-PON, which indicates that XG-PON is more sensitive to the LNA noise. For WLGs ranging from $-56.1 \mathrm{~dB}$ to $-35.7 \mathrm{~dB}$ the transmission of $\mathrm{G}-\mathrm{PON}$ is severely affected by both the PD and the LNA noise. In XG-PON, this region spreads between -42.3 and $-26.7 \mathrm{~dB}$. At WLGs above $-35.7 \mathrm{~dB}(-26.7 \mathrm{~dB})$ the G-PON (XG-PON) NF is mainly affected by the PD noise.

The data presented in Fig. 7 can be used as a look-up table or, alternatively, a piecewise regression can be performed to facilitate 


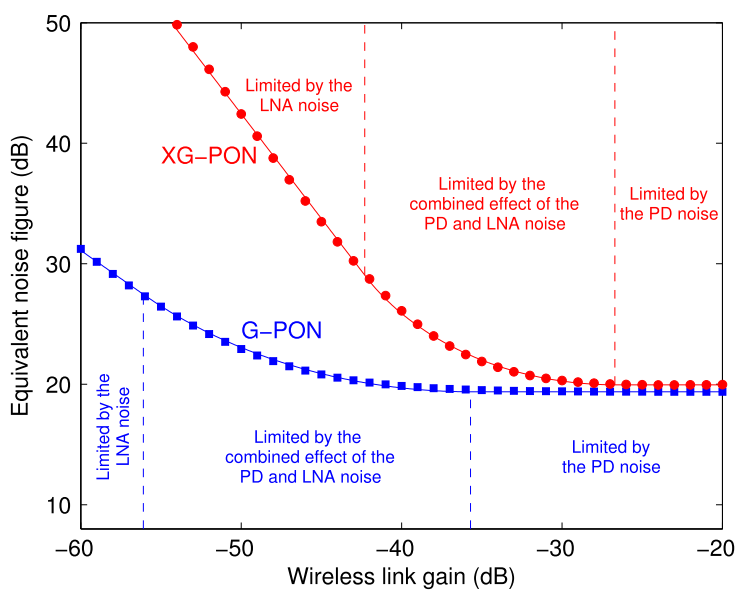

Fig. 7. Equivalent noise figure in terms of the wireless link gain for G-PON and XG-PON.

its use. Eq. (4) and Eq. (5) present the regressions for G-PON and XG-PON, respectively.

$$
\begin{aligned}
\mathrm{NF}_{G} & = \begin{cases}-0.933 \cdot \mathrm{WLG}-24.88 & \mathrm{WLG} \leq-56.1 \\
\sqrt{30.11^{2}-(\mathrm{WLG}+35.57)^{2}}+49.47 & -56.1 \leq \mathrm{WLG} \leq-35.7 \\
19.37 & -35.7 \leq \mathrm{WLG}\end{cases} \\
\mathrm{NF}_{X G} & = \begin{cases}-1.7388 \cdot \mathrm{WLG}-44.49 & \mathrm{WLG} \leq-42.3 \\
\sqrt{17.95^{2}-(\mathrm{WLG}+26.65)^{2}}+37.95 & -42.3 \leq \mathrm{WLG} \leq-26.7 \\
19.95 & -26.7 \leq \mathrm{WLG}\end{cases}
\end{aligned}
$$

\section{Conclusions}

A mm-wave bridge based RF frequency doubling is proposed to overcome accidental fiber cuts in optical access networks. Simulation carried out in VPI Transmission Maker revealed that the photonic mm-wave bridge is capable of converting conventional PON signals at 2.5 and $10 \mathrm{Gbps}$ bitrates and transmit them wirelessly at $81-86 \mathrm{GHz}$ and $102-109.5 \mathrm{GHz}$ keeping a BER lower than $10^{-9}$ provided that the WLG is higher than -55 and $-35 \mathrm{~dB}$, respectively. Results also show that when using the proposed scheme, the closer the fiber cut to the ONU is, the more the system performance is deteriorated.

\section{Acknowledgments}

This work has been partially funded by FAPESP under grant 2015/04113-0 and CONACyT. The work of Dr. Elias Giacoumidis was emanated from EU Horizon 2020 research and innovation programme under the Marie Skłodowska-Curie grant agreement No. 713567 and in part by a research grant from Science Foundation Ireland (SFI) and is co-funded under the European Regional Development Fund under Grant Number 13/RC/2077.

\section{References}

[1] J.M. Simmons, Optical Network Design and Planning, second ed., Springer, 2014

[2] T. Koonen, Trends in optical access and in-building networks, in: Proceedings of European Conference on Optical Communications, 2008, pp. 1-31. http: //dx.doi.org/10.1109/ECOC.2008.4729555.

[3] E. Trojer, S. Dahlfort, D. Hood, H. Mickelsson, Current and Next-Generation PONs: A Technical Overview of Present and Future PON Technology, Tech. Rep., Ericsson, 2008, pp. 64-69.

[4] ITU-T Rec. G.984 series: Gigabit-capable passive optical networks, G-PON, International Telecommunication Union, Tech. Rep. 45(3) (2008) S17-S25. http://dx.doi.org/10.1109/MCOM.2007.344582.
[5] ITU-T Rec. G.987 series: 10-Gibabit-capable passive optical networks (XGPON), International Telecommunication Union, Tech. Rep. 79(3) (2012) 117122.

[6] Y.Sambo, M. Shakir, K. Qaraqe, E. Serpedin, M. Imran, Expanding cellular coverage via cell-edge deployment in heterogeneous networks: Spectral efficiency and backhaul power consumption perspectives, IEEE Commun. Mag. 52 (6) (2014) 140-149. http://dx.doi.org/10.1109/MCOM.2014.6829956.

[7] K. Kitayama, et al., High-speed optical and millimeter-wave wireless link for disaster recovery, in: Globecom Workshops, GC Wkshps, 2015.

[8] T. Kawanishi, Transparent waveform transfer for resilient low-latency links, IEEE Photonics Soc. Newsl. 28 (4) (2014) 4-8.

[9] D. Zhou, S. Subramaniam, Survivability in optical networks, IEEE Netw. 14 (6) (2000) 16-23. http://dx.doi.org/10.1109/65.885666.

[10] J. Chen, L. Wosinska, Analysis of protection schemes in PON compatible with smooth migration from TDM-PON to hybrid WDM/TDM-PON, J. Opt. Netw. 6 (5) (2007) 514-526. http://dx.doi.org/10.1364/JON.6.000514.

[11] C. Mas-Machuca, J. Chen, L. Wosinska, PON protection architectures achieving total cost reduction, in: Proceedings of Asia Communications and Photonics, 2010, pp. 707-708. http://dx.doi.org/10.1109/ACP.2010.5682700.

[12] S. Koenig, J. Antes, D. Lopez-Diaz, I. Kallfass, T. Zwick, C. Koos, W. Freude, J. Leuthold, High-speed wireless bridge at $220 \mathrm{GHz}$ connecting two fiber-optic links each spanning up to $20 \mathrm{~km}$, in: Proceedings of Optical Fiber Communication, OFC, 2012, pp. 1-3.

[13] A. Lebedev, X. Pang, J. Vegas Olmos, M. Beltran, R. Llorente, S. Forchhammer, I. Tafur Monroy, Feasibility study and experimental verification of simplified fiber-supported 60-GHz picocell mobile backhaul links, IEEE Photonics J. 5 (4) (2013) 7200913-7200913. http://dx.doi.org/10.1109/JPHOT.2013.2277011.

[14] Z. Pi, F. Khan, An introduction to millimeter-wave mobile broadband systems, IEEE Commun. Mag. 49 (6) (2011) 101-107. http://dx.doi.org/10.1109/MCOM. 2011.5783993.

[15] J. Beas, G. Castañón, I. Aldaya, G. Campuzano, Aragón-Zavala, Millimeter-wave frequency radio over fiber: A survey, IEEE Commun. Surv. Tutor. PP (99) (2013) 1-27. http://dx.doi.org/10.1109/SURV.2013.013013.00135.

[16] Z. Jia, J. Yu, G. Ellinas, G.K. Chang, Key enabling technologies for opticalwireless networks: Optical millimeter-wave generation, wavelength reuse, and architecture, J. Lightwave Technol. 25 (11) (2007) 3452-3471. http://dx. doi.org/10.1109/JLT.2007.909201.

[17] J.M. Tang, R.P. Giddings, X.Q. Jin, J.L. Wei, X. Zheng, E. Giacoumidis, E. HuguesSalas, Y. Hong, C. Shu, J. Groenewald, K. Muthusamy, Real-time optical OFDM transceivers for PON applications, in: Proceedings of Optical Fiber Communication Conference and, OFC, 2011, pp. 1-3.

[18] G.P. Agrawal, Fiber-Optic Communication Systems, John Wiley and Sons, Inc., 2002.

[19] G. Agrawal, Nonlinear Fiber Optics, third ed., Academic Press, San Diego, CA, 2001.

[20] [Online], Available from: http://www.millitech.com.

[21] [Online], Available from: http://www.hhi.fraunhofer.de.

[22] [Online], Available from: https://www.finisar.com.

[23] S. Saunders, Aragón-Zavala, Antennas and Propagation for Wireless Communication Systems, second ed., Wiley \& Sons, 2007.

[24] [Online], Available from: http://quinstar.com.

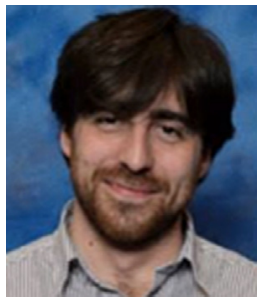

Ivan Aldaya received the B.E. in Telecommunication from the Public University of Navarre (UPNA), Pamplona, Navarre, in 2006. In 2013 received the Ph.D. degree in Communications and Information Technologies from the Instituto Tecnologico y de Estudios Superiores de Monterrey. During his Ph.D., he spent a short research stay of two months in the University of Bologna and a 1-year stay at Telecom Paristech. His main areas of research are radio over fiber networks, optical injection locking of semiconductor lasers, and silicon photonics.

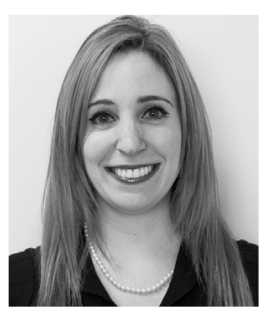

Carolina was born in Medellin, Colombia. She has a Ph.D. in Information Technology and Communications with a doctoral dissertation: Design, implementation and comparison of a new routing protocol for Wireless Sensor Networks. She studied a Master in Science of electronic engineering (Telecommunications) with a thesis named Development of a P2P network with DNS security. Carolina has a Bachelor in electronic engineering with a thesis in Design and construction of a photon counting systems. Carolina's main skills in to apply and develop in the areas of telecommunications, specifically wired and wireless networks, programming and security. 


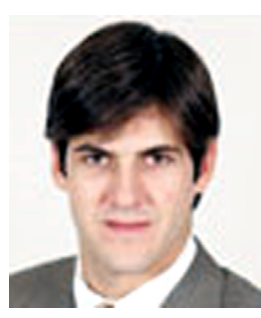

Gabriel Campuzano is an assistant professor at the Department of Electrical and Computational Engineering at Tecnológico de Monterrey since august 2003. He received his undergraduate degree as an Industrial Physics Engineer from the same University (Tecnológico de Monterrey), Campus Monterrey, and holds a M.Sc. (DEA) in Microwaves and Optoelectronics from the "Université de Pierre et Marie Curie", France. He then received the Ph.D. degree in Optical Communications from the "École Nationale Supérieure des Telécommunications" (Telecom ParisTech), France. His research experience includes participation in different projects in the information and communication technology fields with Alcatel Opto+, European Union project OPTIMIST thematic network, and the Government of the State of Nuevo Leon. His fields of interest range in Radio over Fiber Systems for wireless access networks, including mobility in picocells, novel optoelectronic functions, and quantum cryptographic systems for telecommunication wavelengths.

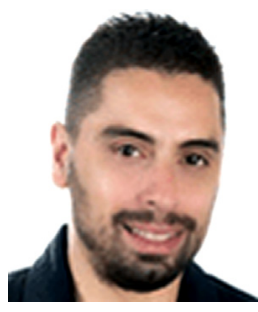

E. Giacoumidis is a Marie-Curie Fellow at Dublin City University \& SFI CONNECT Research Centre of Ireland. His project tackles the "capacity crunch" in optical fiber communications (EPIC: Energy-efficient and Phase-Insensitive Coherent Communications). He has previously worked for various prestigious optical communications research groups: Heriot-Watt University, University of Sydney CUDOS (deputy project leader), Aston University, Bangor University (Ph.D. scholarship), Athens Information Technology, and Telecom-ParisTech, where he was also teaching optical system modelling. He has authored/co-authored more than 100 papers that appeared in international peer-reviewed journals and top conferences (including an invited paper at IEEE/OSA Journal of Lightwave Technology,JLT, and two prestigious postdeadline papers at OSA ACOFT' 15 and IEEE/OSA CLEO Pacific-Rim'17). His research involves balanced theoretical and experimental exploration in high-capacity optical transmission systems with specialization in key modern digital signal processing modulation techniques (OFDM, CAP, PAM-4 etc.) and nonlinear photonics (e.g. Brillouin filtering and amplification) for nextgeneration local, access networks and flexible long-haul optical communications. Dr Giacoumidis is the principal investigator of the world's-first direct-detected optical Fast-OFDM transmission system. He was the first to implement digitalbased machine learning in optical communications (coherent optical OFDM) for fiber nonlinearity compensation. Dr Giacoumidis was nominated an outstanding reviewer of 2016 for IEEE/OSA JLT.

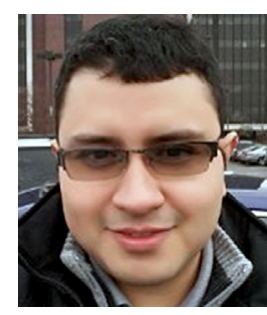

Rafael González received his Ph.D. degree in Telecommunications from Instituto Tecnológico y de Estudios Superiores de Monterrey (ITESM), Monterrey, Mexico. In 2015, he started working as a Test Engineer in Motorola Solutions Inc. His research interests include RF and optical communications as well as computational electromagnetics and instrumentation.

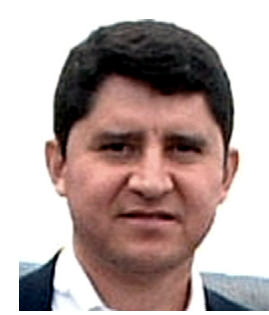

Gerardo Castañón Full professor of Electrical and Computer Engineering at Tecnologico de Monterrey (ITESM). Visiting scientist at the Research Laboratory of Electronics at MIT from August 2015 to July 2016, working on silicon photonics integration and the development of advanced telecommunication photonic devices and sensors in CMOS and beyond-CMOS materials. Member of the national research system in Mexico. Dr. Castanon received the Bachelor of Science in Physics Engineering from the Monterrey Institute of Technology and Higher Education (ITESM), Mexico in 1987. He received the Master of Science degree in Physics (Optics) from the Ensenada Research Centre and Higher Education Mexico in 1989. He also received the Master and Ph.D. degrees in Electrical and Computer Engineering from the State University of New York (SUNY) at Buffalo in 1995 and 1997 respectively. He was supported by the Fulbright scholarship through his Ph.D. studies. From January of 1998 to November of 2000 he was a Research Scientist working with Alcatel USA Corporate Research Center in Richardson TX. Where he was doing research on IP over WDM, dimensioning and routing strategies for next generation optical networks and the design of all-optical routers. From December of 2000 to August of 2002 he was a senior researcher with Fujitsu Network communications doing research on ultra high speed transmission systems. Dr Castanon has over 100 publications in journals and conferences and 4 international patents. He frequently acts as a reviewer for IEEE journals. He is a Senior member of the IEEE Communications and Photonics societies. He is a member the Academy of Science in Mexico. 